\title{
Highly sensitive polymer-based cantilever-sensors for DNA detection
}

Ultramicroscopy 105, 215 (2005), original manuscript

M. Calleja ${ }^{\# 1,2}$, M. Nordström² ${ }^{2}$ M. Álvarez ${ }^{1}$, J. Tamayo ${ }^{1}$, L. M. Lechuga ${ }^{1}$, A. Boisen ${ }^{2}$

${ }^{1}$ Biosensors Group, Nacional Center of Microelectronics (CNM-CSIC), Isaac Newton 8, Tres Cantos, E-28760 Madrid, Spain

${ }^{2}$ Mikroelektronics Centret, Technical University of Denmark, 345E, DK-2800, Lyngby, Denmark

\#Corresponding author. E-mail: mcalleja@imm.cnm.csic.es

\begin{abstract}
We present a technology for the fabrication of cantilever arrays aimed to develop an integrated biosensor micro-system. The fabrication process is based on spin coating of the photosensitive polymer and near-ultraviolet exposure. Arrays of up to 33 microcantilevers are fabricated in the novel polymer material SU-8. The low Young's modulus of the polymer, 40 times lower than that of silicon, enables to improve the sensitivity of the sensor device for target detection. The mechanical properties of SU-8 cantilevers, such as spring constant, resonant frequency and quality factor are characterized as a function of the dimensions and the medium. The devices have been tested for measurement of the adsorption of single stranded DNA and subsequent interstitial adsorption of lateral spacer molecules. We demonstrate that sensitivity is enhanced by a factor of six compared to that of commercial silicon nitride cantilevers.
\end{abstract}

Keywords: biosensors, micromechanical sensors, DNA, polymer cantilevers 
PACS: 87.80.-y, 87.17.Gg, 07.10.Cm, 87.83.+a 


\section{Introduction}

Recent advances in microfabrication technologies have triggered new applications for micro/nano-tools. Microcantilevers, such as those used in Atomic Force Microscopes, have been recently employed as a new class of biosensors[1,2]. The so-called nanomechanical biosensors have demonstrated that they are capable of detecting singlebase mismatches in oligonucleotide hybridization[2] as well as performing protein recognition[3,4] with extreme sensitivity. Among the advantages of nanomechanical biosensors are the potential for performing local, high resolution and label-free molecular recognition measurements on a portable device. Also, the reduced sensor area allows drastic decrease of the reagent consumption.

The working principle for nanomechanical biosensors relies on the induced surface stress that arises when molecules bind to a surface[5]. When a monolayer of molecules is immobilized on one side of the cantilever, a cantilever deflection results from the differential surface stress between opposite sides of the cantilever.

The cantilever deflection, $\Delta \mathrm{z}$, resulting from this difference in surface stress can be approximated by[6],

$$
\Delta z \cong \frac{3(1-v) L^{2}}{E t^{2}}\left(\Delta \sigma_{1}-\Delta \sigma_{2}\right)
$$

where $\mathrm{E}$ is the Young's modulus, $v$ is Poisson ratio, $\mathrm{t}$ and $\mathrm{L}$ are thickness and length of the beam and $\Delta \sigma_{1}-\Delta \sigma_{2}$ accounts for the differential surface stress. 
Usually, cantilever deflection is measured by the optical leverage technique but also other alternative read-out methods have been used, such as interferometric detection[7] and integrated piezoresistive read-out[8,9]. Usually, silicon or silicon nitride cantilevers, as those designed for AFM imaging, are employed both in the static[2-5,7-10] and dynamic modes[11,12]. Most commonly, cantilevers are disposed after each measurement. Hence, a fabrication technology capable of obtaining highly sensitive devices while production costs and fabrication time are kept low, would be very valuable for the future development of sensors for point-of-care applications or environmental control devices[4].

Here we present a technology for the fabrication of polymeric cantilevers to be used as biochemical sensors. The fabrication process is based on spin coating of a photosensitive polymer and near-ultraviolet exposure. It does not require any silicon moulds or long $\mathrm{KOH}$ etching steps, since cantilever biosensors do not need a tip, as opposed to cantilevers for AFM imaging[13].

The cantilever fabrication process presented is inexpensive, fast and reliable. Also, The polymer fabrication technique provides a convenient way to realize arrays of multiple sensors and to integrate them into a miniaturized biochemical analysis system[8,14].

The feasibility of the application of a polymeric cantilever for biological detection is demonstrated by measuring the immobilization process of thiolated single stranded DNA (ssDNA) on a gold-coated cantilever. The response of SU-8 cantilevers is 
compared to that of commercial silicon nitride cantilevers. The high sensitivity of polymeric cantilevers envisions the development of sensitive DNA nanomechanical chips that could be integrated with microfluidics for sample delivery, providing fast and sensitive biosensors for real time measurements.

\section{Materials and reagents}

The sensors presented in this work are fabricated in the polymer SU-8 (MicroChem Corp.)[15,16]. SU-8 is an epoxy-based photoresist. It has a high chemical resistance, which makes it a suitable component material. The resist is compatible with conventional microfabrication techniques and it is relatively easy and fast to process. The use of SU-8 polymer has recently become interesting for the fabrication of passive components such as microfluidic devices[14]. Also, AFM probes[13] and cantilevers with integrated strain gauge readout in a microliquid system[8] have been fabricated in SU-8.

DNA oligonucleotides were commercially prepared by Integrated DNA Technologies. The DNA probes were 12 nucleotides in length and synthesized with a thiol linker group (HS- $\left.\left(\mathrm{CH}_{2}\right)_{6}-\right)$, in order to form a self assembled monolayer onto the cantilever's gold-coated side. The spacer molecule, 6-Mercapto-1-hexanol (MCH), was purchased from Sigma Chemical Co. For MCH treatment, a 1mM solution was prepared and introduced into the liquid cell after oligonucleotides immobilization. Immobilization buffer was prepared with $50 \mathrm{mM}$ phosphate buffered (PB) ph 7.0, from Fluka, and $\mathrm{NaCl}$ $0.5 \mathrm{M}$. 


\section{Measurement set-up}

Cantilever deflection measurements were carried out by using the well-known optical beam deflection method employed in most atomic force microscopes. A laser beam is focused on the back of the cantilever and reflected to a segmented photodiode. The photocurrents from the upper and lower segments are amplified and connected to a data acquisition card (National Intruments) for deflection monitoring in real-time. Acquisition software was programmed in Labview (National Instruments). Cantilever movements are recorded as the current intensity difference between upper and lower segments in the photodiode. Figure 1 shows a photograph of the experimental set-up.

The experiments were carried out in a commercial fluid cell (Digital Instruments, Veeco) with an inlet and outlet for liquid flow. The top part of the fluid cell is made out of glass and the bottom is closed with a coverslip using a rubber O-ring. The configuration of the diode laser, photodetector and fluid cell is shown in figure 1. Flow and injection of sample are performed by using a peristaltic pump (Gilson). The volume enclosed in the fluid cell is about $50 \mu \mathrm{l}$ and the flow rate used in these experiments was $0.3 \mu \mathrm{l} / \mathrm{s}$, approximately. The liquid flow was controlled by an injector valve, which allows switching between continuous buffer solution flow and analyte injection. The system is isolated from mechanical vibrations.

\section{Cantilever design and fabrication}

The cantilever arrays were designed to fit in commercial fluidic headers. Arrays of 9, 15, 17 and 33 cantilevers of different dimensions were fabricated (see table 1). 
Cantilevers were fabricated with different lengths $(100 \mu \mathrm{m}$ and $200 \mu \mathrm{m})$ and widths (20, 30 and $50 \mu \mathrm{m})$. Cantilever thickness was varied from $1.3 \mu \mathrm{m}$ to $2 \mu \mathrm{m}$.

The process sequence for the fabrication of the devices is shown in figure 2. A silicon wafer is used during the process as a support for the multiple SU-8 and metallic layers. First, a layer of $5 \mathrm{~nm} / 50 \mathrm{~nm} / 50 \mathrm{~nm} \mathrm{Cr} / \mathrm{Au} / \mathrm{Cr}$ is deposited onto the wafer (figure 2(a)). This metal layer will be used to release the chips from the wafer in a wet chromium etch. A $1.5 \mu \mathrm{m}$ thick SU-8 layer is spun over the wafer (500 rpm for $5 \mathrm{~s}$, followed by $5000 \mathrm{rpm}$ for $30 \mathrm{~s}$ ) and pre-baked at $90{ }^{\circ} \mathrm{C}$ to evaporate most of the solvent. Cantilever geometry is patterned by UV exposure with a wavelength of $365 \mathrm{~nm}$ at a dose of 450 $\mathrm{mJ} / \mathrm{cm}^{2}$ (figure 2 (b)). The resist is post-baked at $90{ }^{\circ} \mathrm{C}$ for 4 minutes to further promote the crosslinking of UV-exposed SU-8. Afterwards, the remaining non-crosslinked SU-8 is removed by immersion for 2 minutes in an SU-8 developer (propylene glycol monomethyl ether acetate, PGMEA). On top of the SU-8 resist a gold layer is deposited by electron beam evaporation (see figure 2 (c)-(e)) and patterned by lift-off techniques. A thin titanium layer is used to improve the adhesion of gold to SU-8. The gold layer allows reflection of the laser beam, and it is also crucial for surface stress-based bio/chemical detection, in which composition of the opposite surfaces must be different for differential adsorption. Moreover, the gold coating is ideal for strong anchorage of proteins and nucleic acids using self-assembly chemistry. Afterwards, the SU-8 is hard baked at $120{ }^{\circ} \mathrm{C}$ for 1 minute. Finally, a $400 \mu \mathrm{m}$ thick layer of SU-8 is spun onto the wafer to define the support for the cantilever array structure (see figure 2(f)) and the completed devices are released in wet chromium etch (figure 2(g)). Optical images of the finished devices are shown in figure 3. 


\section{Mechanical Characterizacion}

In order to measure the spring constant $(\mathrm{k})$ of the fabricated cantilevers, we used an AFM silicon nitride cantilever (Olympus) which was previously calibrated. In this experimental set-up, the AFM cantilever tip is placed in contact with the free end of the SU-8 cantilever, which is placed on top of a piezoelectric tube that allows precise vertical motion. We measure the deflection of the AFM cantilever, whilst the piezoelectric tube is extended hundreds of nm in cyclic sweeps. Thus, deflection vs. distance curves are obtained, in which the slope depends on the stiffness of the SU-8 cantilever, i.e., softer cantilevers give smaller slopes. The measured elastic constants for different SU-8 cantilevers are shown in table 1.

The dynamic response of the cantilevers was characterized by measuring their Brownian motion produced by the thermal kicking forces. This was characterized by measuring the Fast Fourier Transform (FFT) of the photodetector noise. The resulting spectra for $200 \mu \mathrm{m}$ and $100 \mu \mathrm{m}$ long cantilevers are shown in figure 4 . The resonant peaks were fitted with the damped harmonic oscillator model to determine the resonant frequency and quality factor. We have studied the resonance frequency and the quality factor both in air and liquid environment. In table 1, we present a summary of the studied parameters. The decrease of the resonant frequency and Q when cantilevers are immersed in water, is produced by the drag force generated in liquids[17].

As stated in equation (1), the sensitivity of the cantilever is given by its dimensions and mechanical material properties. The polymer used here has a Young's modulus of about 
$5 \mathrm{GPa}, 40$ times smaller than that of silicon[18]. This allows fabricating cantileversensors with high sensitivity. Also, the dimensions of the cantilevers can be controlled in a very simple way. Cantilever length and width result from the chosen design of the masks for photolithography, but cantilever thickness can be tuned by simply choosing different spinning speeds during polymer deposition. So, even by using the same photolithography masks and in the same batch or even within the same array, cantilevers with different mechanical properties can be realized.

\section{Characterization of DNA immobilization}

The performance of SU-8 cantilevers was tested in the static mode by monitoring the adsorption of thiolated ss-DNA on gold.

Prior to immobilization experiments, cantilevers were cleaned first by rinsing in isopropanol, acetone, ethanol and DI water, in this same sequence, followed by a $10 \mathrm{~s}$ immersion in a mixture of $\mathrm{HCl}$ an $\mathrm{HNO}_{3}$ in proportion 3:1 in volume. This procedure yields a fresh and clean gold surface which is crucial for an efficient thiol immobilization.

To test the performance of the devices, we have injected $20 \mathrm{ml}$ of a $2 \mu \mathrm{M}$ solution of thiolated ss-DNA in buffer solution. The ssDNA is modified with a thiol linker, SH$\left(\mathrm{CH}_{2}\right)_{6^{-}}$, for attachment to the gold-coated side via the covalent bond between the sulfur and gold atoms. The SU-8 cantilever used for this experiment was $200 \mu \mathrm{m}$ long and 1.3 $\mu \mathrm{m}$ thick. 
After injection of $2 \mu \mathrm{M}$ thiolated ss-DNA, the cantilever bends away from the gold coated surface during the formation of the self-assembled monolayer (see figure 5). This indicates that chemisorption of thiolated DNA produces a compressive surface stress. This is in agreement with previous works and it is probably related to the electronic redistribution on gold due to the S/Au bond[19] and to the electrostatic and steric repulsion between the molecular chains. Figure 5 shows the deflection signal during the injection of the sample solution. Cantilever bending after completion of the layer is of $248 \mathrm{~nm}$. Next, a solution of 1mM of 6-mercapto-1-hexanol (MCH) was injected. MCH is a 6-carbon chain molecule terminated at both ends with thiol (-SH) and hydroxyl groups $(-\mathrm{OH})$. This short molecule displaces the weakly adsorbed molecules, i.e. those not covalently bound, like molecules physisorbed through nucleotide chain-gold interactions. Also, since the hydroxyl group does not interact with the nucleotide chain, MCH acts as a spacer molecule between the DNA chains, enhancing the accessibility of ssDNA molecules for hybridization with complementary nucleic acids. From figure 5, a further downward deflection of $109 \mathrm{~nm}$ is observed after injection of $1 \mathrm{mM} \mathrm{MCH}$.

For comparison, we have repeated the same experiment by using a commercial silicon nitride cantilever. This cantilever was $0.8 \mu \mathrm{m}$ thick, $200 \mu \mathrm{m}$ long and $40 \mu \mathrm{m}$ wide, with an elastic constant of $0.11 \mathrm{~N} / \mathrm{m}$. Here DNA immobilization produces a cantilever bending of only $41 \mathrm{~nm}$, while MCH injection deflects the sensor by $15 \mathrm{~nm}$ (see inset in figure 5).

Compared to a silicon nitride commercial cantilever, the cantilever deflection for the immobilization of $2 \mu \mathrm{M}$ thiolated ss-DNA was six times larger for these new polymeric 
probes. The measured values are compatible with equation (1) regarding the material properties of silicon nitride and SU-8 and the actual dimensions for each kind of cantilever in the experiments.

The elastic constant of SU-8 cantilevers $200 \mu \mathrm{m}$ long and $1.3 \mu \mathrm{m}$ thick is about 0.007 $\mathrm{N} / \mathrm{m}$. Fritz et al. have reported that the hybridization of DNA to a target layer gives rise to a surface stress change of $1 \mathrm{mN} / \mathrm{m}$ [2]. According to equation (1) and for an SU-8 cantilever $200 \mu \mathrm{m}$ long and $1.3 \mu \mathrm{m}$ thick, a surface stress change of $1 \mathrm{mN} / \mathrm{m}$ would deflect the cantilever about $11 \mathrm{~nm}$, which can be detected with the optical beam deflection method with a high signal-to-noise ratio. For the same differential surface stress a commercial silicon nitride cantilever as that in the experiments would only deflect $1.2 \mathrm{~nm}$. Considering a minimum detectable deflection of $0.5 \mathrm{~nm}$, surface stress changes as small as $60 \mu \mathrm{N} / \mathrm{m}$ could be detected by SU-8 cantilevers. 


\section{Conclusions}

We have demonstrated that a new kind of cantilevers fabricated in polymer material is suitable for bio/chemical detection. The fabrication process presented provides a fast, inexpensive and reliable method to obtain arrays of cantilevers for bio/chemical measurements. We have successfully measured the adsorption of ssDNA. Also, SU-8 material properties are ideal for obtaining highly sensitive sensors. The cantilevers presented here have demonstrated an improved sensitivity compared to commonly used commercial silicon nitride cantilevers. As an additional advantage, SU-8 resist has proven to be very convenient for the fabrication of arrays of cantilevers that could be applied for multiple target detection experiments. Furthermore, the use of a polymer instead of silicon opens up the possibility of developing new surface chemistry processes for the building of the biosensing layer.

\section{Acknowledgements}

The authors acknowledge P. Rasmussen and L. G. Carrascosa for fruitful discussion. M. C. acknowledges financial support from I3P-CSIC and MERG-CT-2004-510638. J. T. and M. A. acknowledge financial support from MCyT (BIO2001-1235- C03-01 and GEN2001-4856-C13-11). 


\section{References}

[1] R. Raiteri, M. Grattarola, H-J. Butt, P. Skládal, Sensors and Actuators B, 79, 115$126(2001)$

[2] J. Fritz, M. K. Baller, H. P. Lang, H. Rothuizen, P. Vettiger, E. Meyer, H. J. Güntherodt, Ch. Gerber, J. W. Gimzewski, Science 288, 316 (2000)

[3] G. Wu, R. H. Datar, K. M. Hansen, T. Thundat, R. J. Cote, A. Majumdar, Nature Biotechnology, 19, 856-860 (2001)

[4] M. Alvarez, A. Calle, J. Tamayo, L. M. Lechuga, A. Abad, A. Montoya, Biosensors and Bioelectronics, 18, 649 (2003)

[5] G. Wu, H. Ji, K. Hansen, T. Thundat, R. Datar, R. Cote, M. F. Hagan, A. K. Chakraborty, A. Majumdar, Proc. Natl. Acad. Sci. USA 98, 1560 (2001)

[6] J. E. Sader, Journal of Applied Physics 89, 2911 (2001)

[7] C. A. Savran, T. P. Burg, J. Fritz, S. R. Manalis, Applied Physics Letters 83, 1659$1661(2003)$

[8] M. Calleja, P. Rasmussen, A. Johansson, A. Boisen, Proceedings of the SPIE - The International Society for Optical Engineering, Vol. 5116, 314 (2003)

[9] P.A. Rasmussen, J. Thaysen, O. Hansen, S.C. Eriksen, A. Boisen , Ultramicroscopy 97, 371 (2003)

[10] R. McKendry, J. Zhang, Y. Arntz, T. Strunz, M. Hegner, H. P. Lang, M. K. Baller, U. Certa, E. Meyer, H-J, Güntherodt, Ch. Gerber, Proc. Natl. Acad. Sci. 99, 9783-9788 (2002)

[11] J. Tamayo, A. D. Humphris, A. M. Malloy, M. J. Milles, Ultramicroscopy 86, 167$173(2001)$ 
[12] J. Tamayo, M. Alvarez, L. M. Lechuga, Sensors and Actuators B, 6842, 1-7 (2003)

[13] G. Genolet, J. Brugger, M. Despont, U. Drechsler, P. Vettiger, N. F. Rooij, D. Anselmetti, Rev. Sci. Instrum. 70, 2398 (1999)

[14] M. O. Heuschkel, L. Guerin, B. Buisson, D. Bertrand, P. Renaud, Sensors and Actuators B 48, 356 (1998)

[15] H. Lorenz, M. Despont, N. Fahrni, N. Labianca, P. Renaud, P. Vettiger, J. Micromech. Microeng. 7, 121 (1997)

[16] H. Lorenz, M. Despont, N. Fahrni, J. Brugger, P. Vettiger, P. Renaud, Sensors Actuators A 64, 33 (1998)

[17] J. Tamayo, A. D.L. Humphris, M. J. Miles, Applied Physics Letters 77, 582 (2000)

[18] R. Feng, R. J. Farris, Journal of Micromechanical Microengeneering 13, 80-88 (2003)

[19] M.Álvarez, L.G.Carrascosa, J.Tamayo, A.Calle and L.M. Lechuga, Proceedings of SPIE, 5118 197- 206 (2003) 


\section{Table captions}

Table 1. Design parameters for the cantilever arrays. Measured elastic constant and resonant frequency for the different cantilevers are shown. Thermal noise signal in the photodetector is measured to determine the resonance frequency both in air and water. The quality factor measured in air and liquid is also presented. 


\section{Figure captions:}

Figure 1. Picture of the experimental set-up. A diode laser is focused on the cantilevers which are enclosed in the liquid cell. The reflected beam is measured by a photodetector.

Figure 2. Fabrication process sequence. (a) Evaporation of release layer $(\mathrm{Cr} / \mathrm{Au} / \mathrm{Cr}$ $(5 \mathrm{~nm} / 50 \mathrm{~nm} / 50 \mathrm{~nm}))$. (b) Spinning and patterning of first layer of SU-8 to define the cantilevers. (c) , (d) and (e) Lift-off lithography of the metallic layer (Ti/Au) for immobilization of biomolecules and laser reflection. (f) Patterning of the support layer on $400 \mu \mathrm{m}$ thick SU-8. (g) Release of finished structure by wet etching.

Figure 3. Optical image of finished devices. (a) Array of 17 cantilevers. The cantilever length is $200 \mu \mathrm{m}$ and their width is $20 \mu \mathrm{m}$. The distance between consecutive cantilevers (center to center) is $100 \mu \mathrm{m}$. (b) Cantilever array of $100 \mu \mathrm{m}$ long and $20 \mu \mathrm{m}$ wide cantilevers.

Figure 4. (a) Dynamic response of a cantilever $200 \mu \mathrm{m}$ long and $50 \mu \mathrm{m}$ wide in air. Resonance frequency $=15.3 \mathrm{kHz}$ and $\mathrm{Q}=12.8$. (b) Dynamic response of the cantilever in (a) when immersed in water. Resonance frequency $=2.3 \mathrm{kHz}$ and $\mathrm{Q}=1.3$. (c) Dynamic response of a cantilever $100 \mu \mathrm{m}$ long and $20 \mu \mathrm{m}$ wide in air. Resonance frequency $=40.4 \mathrm{kHz}$ and $\mathrm{Q}=15.6$. (d) Dynamic response of the cantilever in (c) when immersed in water. Resonant frequency $=7.3 \mathrm{kHz}$ and $\mathrm{Q}=1.4$. The resonant peaks were fitted with the damped harmonic oscillator model to determine the resonant frequency and quality factor. 
Figure 5. Adsorption of $2 \mu \mathrm{M}$ thiolated ss-DNA on a gold-coated SU-8 cantilever. The cantilever is $1.3 \mu \mathrm{m}$ thick and $200 \mu \mathrm{m}$ long. DNA is immobilized through sulfur groups on the gold coated side of the cantilever. Next, $1 \mathrm{mM}$ solution of $\mathrm{MCH}$ is also injected. The compressive surface stress on the gold coated side produces the bending of the cantilever. The inset shows the same experiment but performed with a commercial silicon nitride cantilever $0.8 \mu \mathrm{m}$ thick and $200 \mu \mathrm{m}$ long. The deflection signal is six times larger for the measurement performed with the SU-8 cantilever. 
Calleja et al.

Table 1

\begin{tabular}{|c|c|c|c|c|c|c|c|}
\hline $\begin{array}{c}\text { Length } \\
(\mu \mathrm{m})\end{array}$ & $\begin{array}{c}\text { Width } \\
(\mu \mathrm{m})\end{array}$ & $\begin{array}{c}\text { Thickness } \\
(\mu \mathrm{m})\end{array}$ & $\begin{array}{c}\mathbf{K} \\
(\mathrm{N} / \mathrm{m})\end{array}$ & $\begin{array}{c}\mathbf{f}_{\text {air }} \\
(\mathrm{kHz})\end{array}$ & $\begin{array}{c}\mathbf{f}_{\text {liquid }} \\
(\mathrm{kHz})\end{array}$ & $\mathbf{Q}_{\text {air }}$ & $\mathbf{Q}_{\text {liquid }}$ \\
\hline $200 \pm 1$ & $20 \pm 1$ & $1.5 \pm 0.1$ & $0.0245 \pm 0.0011$ & 17 & 3.4 & 15.3 & 1.6 \\
\hline $200 \pm 1$ & $50 \pm 1$ & $1.7 \pm 0.1$ & $0.0640 \pm 0.0015$ & 15 & 2.3 & 12.8 & 1.3 \\
\hline $100 \pm 1$ & $20 \pm 1$ & $1.5 \pm 0.1$ & $0.286 \pm 0.017$ & 40 & 7.3 & 15.6 & 1.4 \\
\hline
\end{tabular}


Calleja et al.

Figure 1

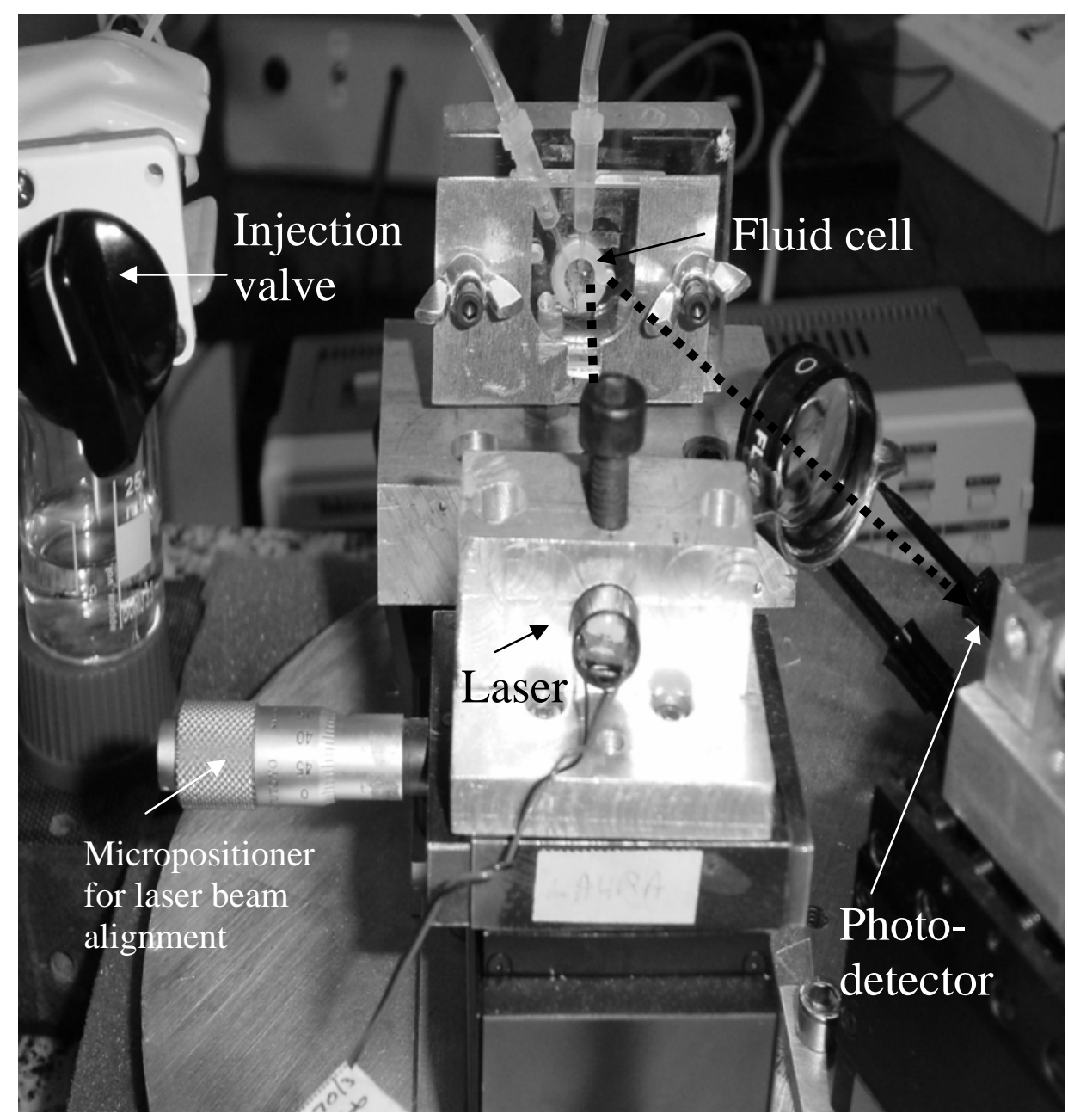


Calleja et al.

Figure 2

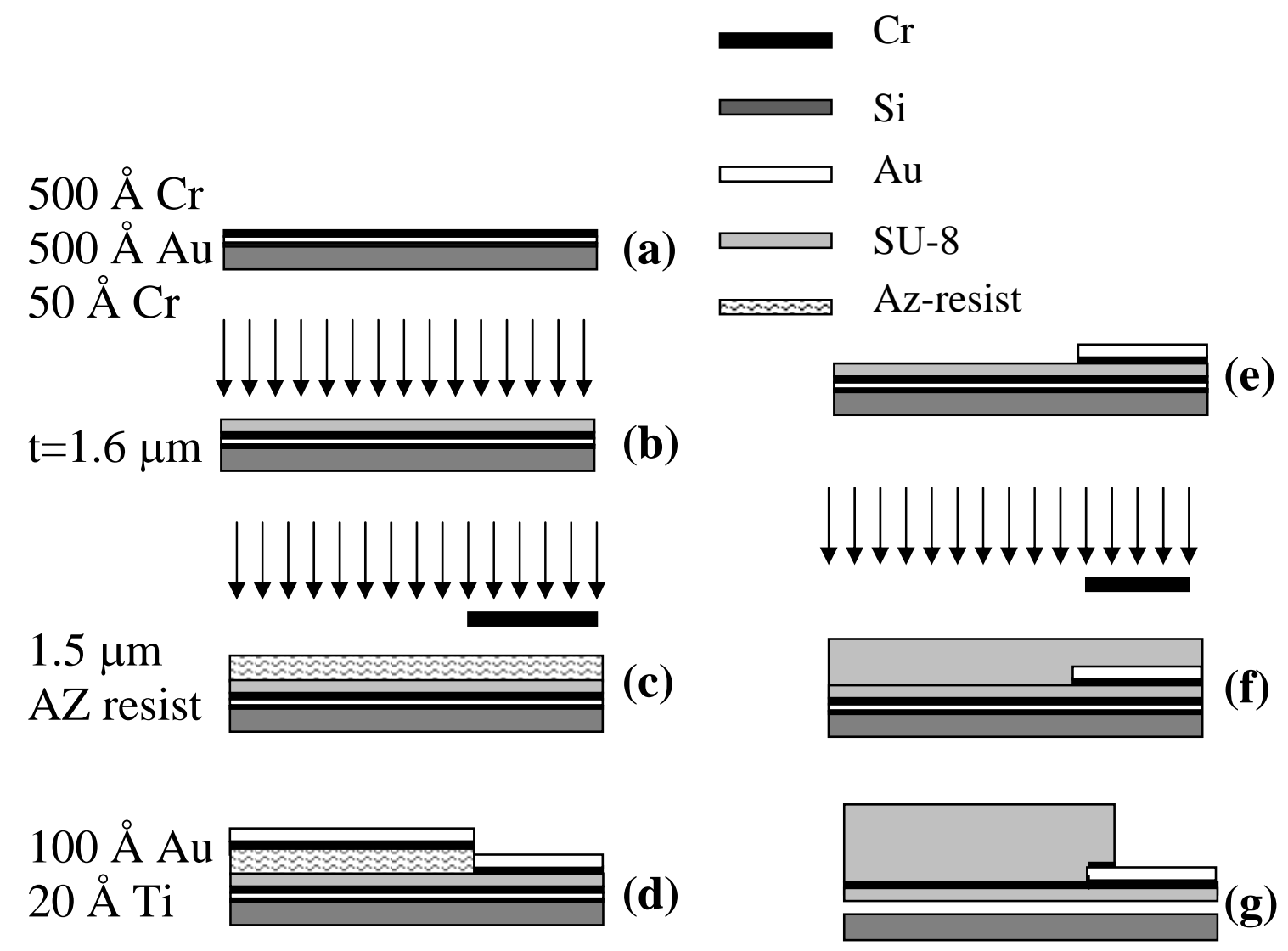


Calleja et al.

Figure 3

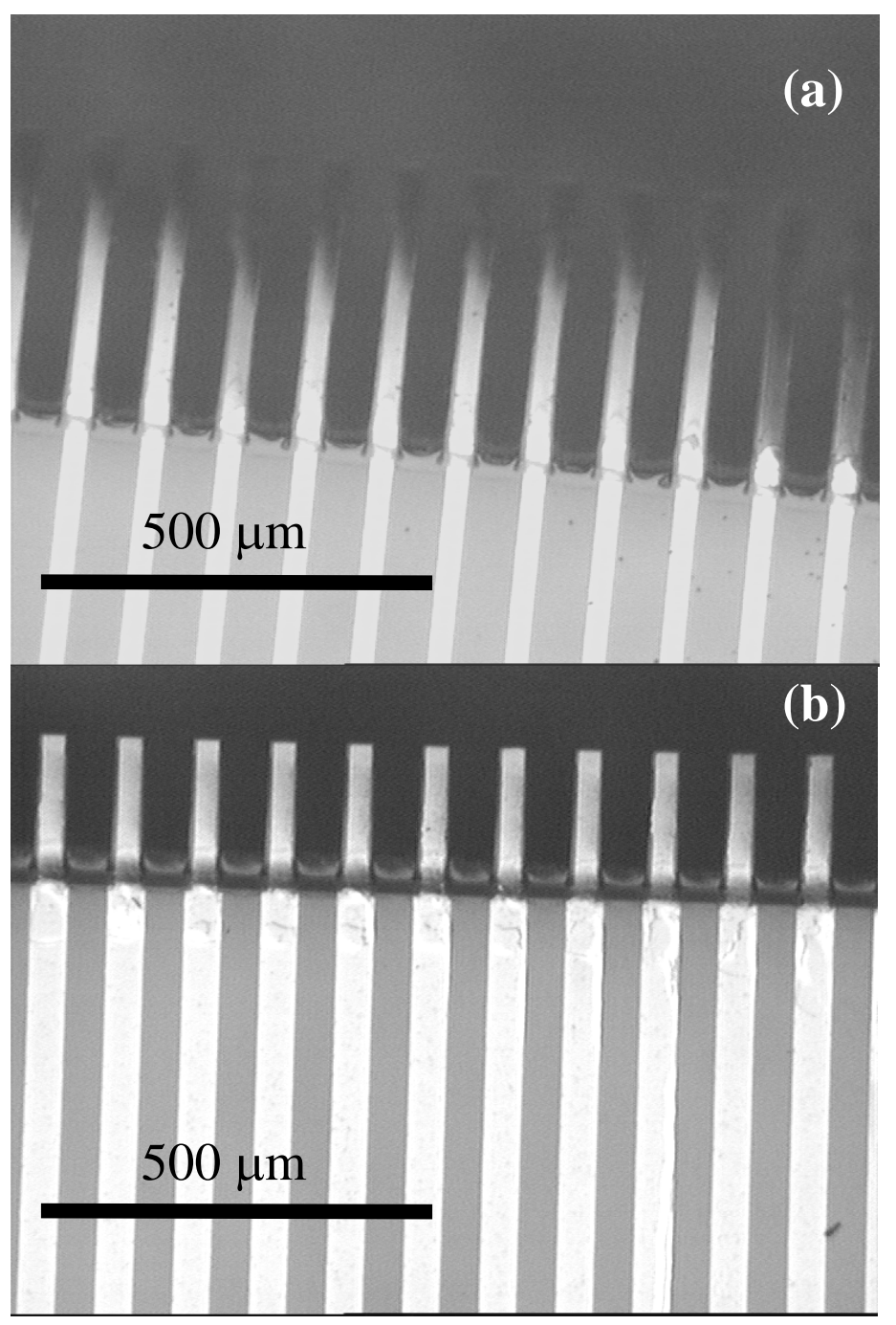


Calleja et al.

Figure 4

(a)

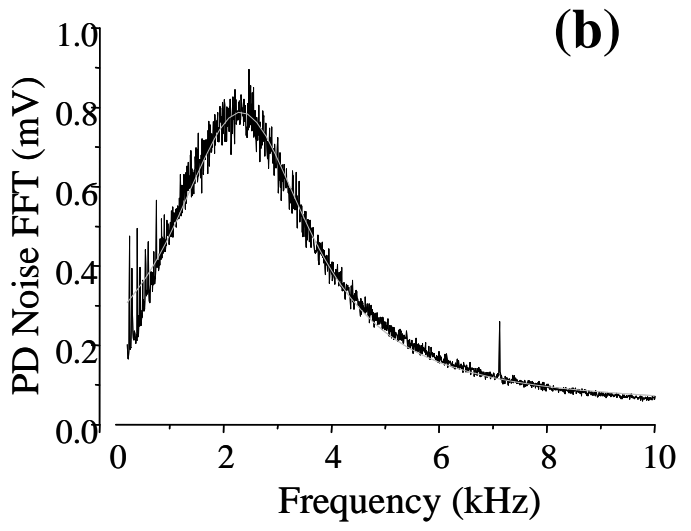

(c)
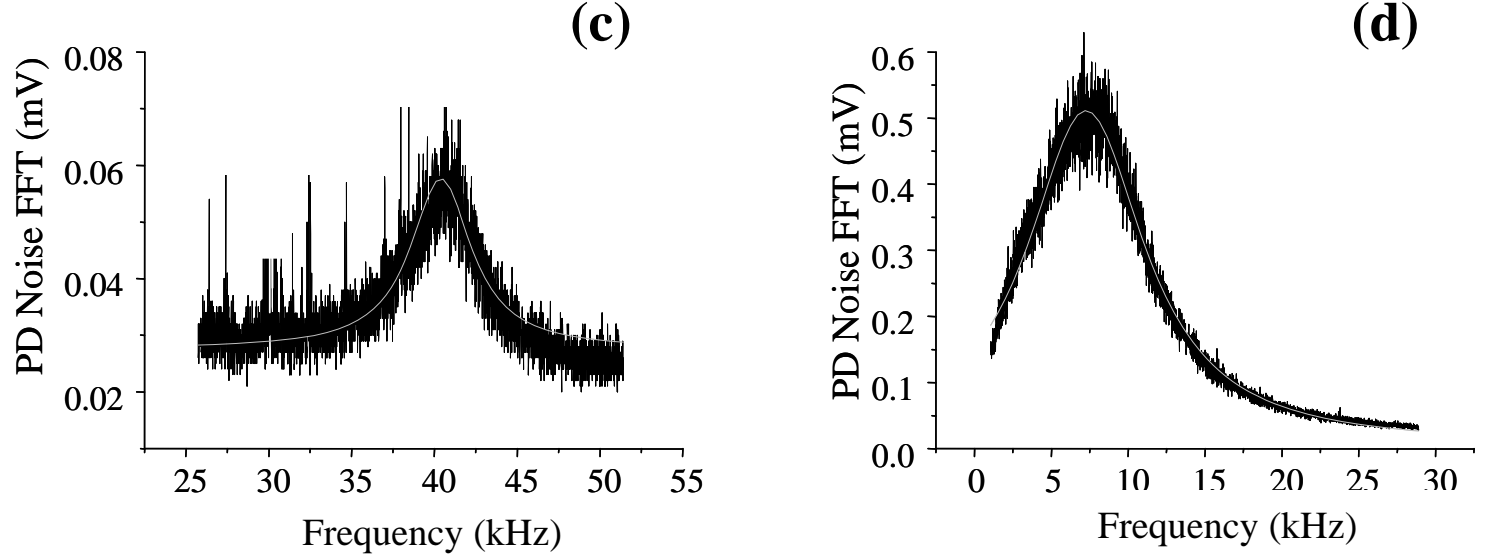
Calleja et al.

Figure 5

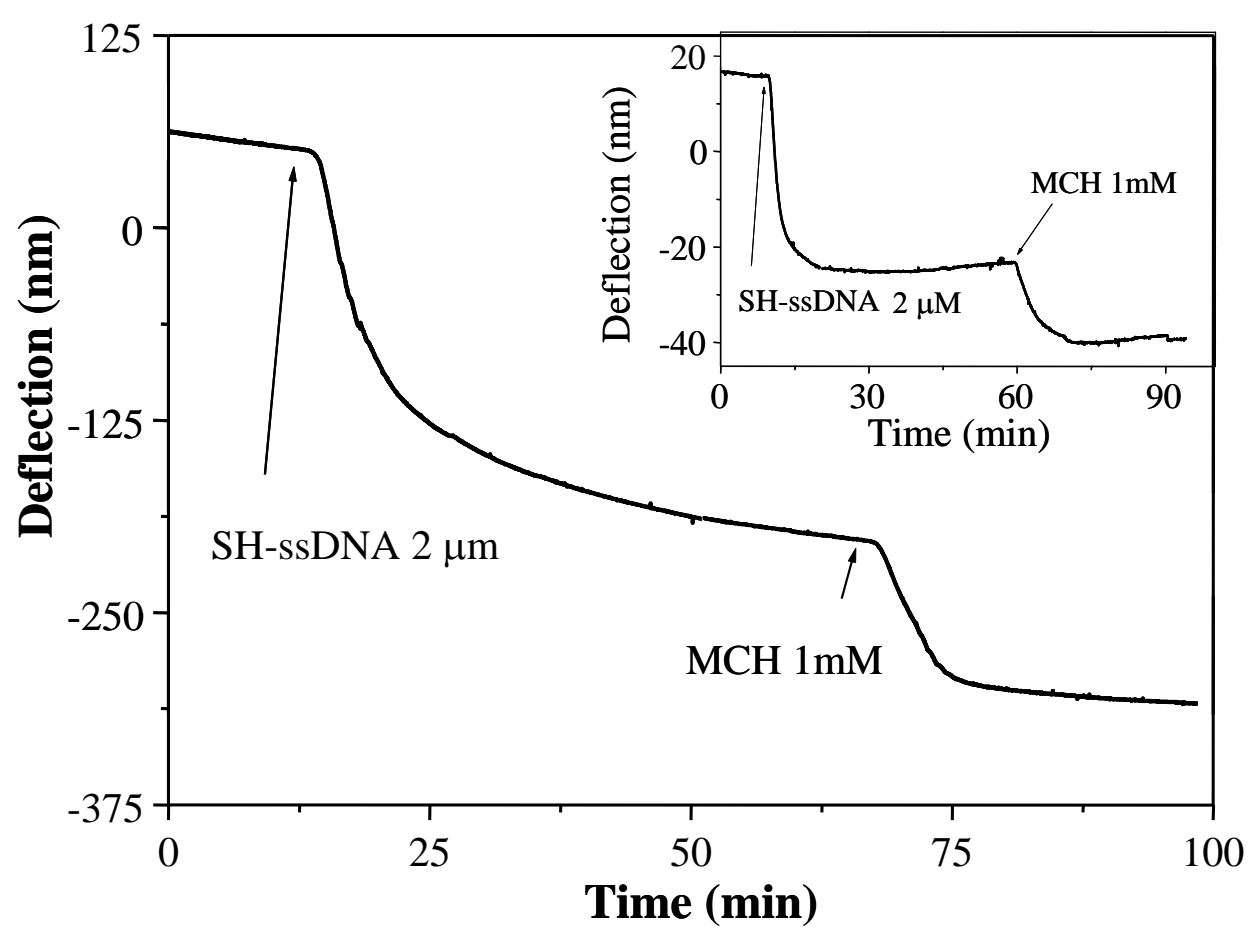

